\title{
Candesartan Mediated Amelioration of Cisplatin-Induced Testicular Damage Is Associated with Alterations in Expression Patterns of Nephrin and Podocin
}

\author{
Noritoshi Enatsu, Hideaki Miyake, Koji Chiba, and Masato Fujisawa \\ Division of Urology, Kobe University Graduate School of Medicine, 7-5-1 Kusunoki-cho, Chuo-ku, Kobe 650-0017, Japan \\ Correspondence should be addressed to Noritoshi Enatsu; nenatsu@med.kobe-u.ac.jp
}

Received 14 May 2015; Accepted 30 July 2015

Academic Editor: Rafael Fissore

Copyright (C) 2015 Noritoshi Enatsu et al. This is an open access article distributed under the Creative Commons Attribution License, which permits unrestricted use, distribution, and reproduction in any medium, provided the original work is properly cited.

\begin{abstract}
Nephrin and podocin are known to be closely related to the pharmacological effects of angiotensin-II receptor blocker (ARB). The objectives of this study were to investigate the role of nephrin and podocin using cisplatin-induced testicular damage and to evaluate the effect of ARB. At first, we evaluated the effects of cisplatin either alone or in combination with ARB candesartan on changes in expression patterns of nephrin and podocin in the rat testes. We then conducted in vitro studies to investigate the effects of angiotensin using cultured Sertoli cells, line TM4. As a result, the expression of nephrin and podocin was shown to localize around the basal membrane of seminiferous tubules. Treatment with cisplatin resulted in a marked decrease in the expression of nephrin and podocin and induced a shift of both proteins from linear to granular expression patterns, accompanying the increased apoptotic index in the testes; these changes were partially restored by the additional administration of candesartan. In vitro studies with TM4 revealed the angiotensin-II mediated expression changes of nephrin and podocin. These findings suggest that candesartan can prevent cisplatin-induced testicular damage by regulating expression patterns of the nephrin-podocin complex in the testes.
\end{abstract}

\section{Introduction}

Nephrin and podocin are the well-known proteins, which are connected to each other and play an important role in maintaining the function of the slit diaphragm, a structure likely to be the filtration barrier of the glomerular capillary wall as a highly developed cell-cell junction [1]. Furthermore, several recent studies have reported that the development of proteinuria, not only in congenital nephrotic syndrome but also in acquired renal diseases, is regulated, at least in part, by renal slit diaphragm proteins, including nephrin and podocin [2].

To date, there have been a number of studies demonstrating the involvement of disorders of cell-cell junctions in the development of pathological conditions in the testes [3]. We previously reported the association between impaired spermatogenesis and disorganization of major components of tight junctions in Sertoli cells in both experimental and clinical studies $[4,5]$. Collectively, these findings indicate that it might be interesting to investigate whether testicular function is regulated by nephrin and podocin, like some other molecules mediating pathophysiological roles in cellcell junctions in the testes. In fact, the expression of nephrin and podocin was confirmed in murine testes, and Sertoli cells were suggested to be the testicular origin of these proteins $[6,7]$. Furthermore, there have been several studies focusing on angiotensin-II mediated kidney damage through nephrin and podocin. For example, angiotensin-II receptor blocker (ARB) is reported to have antiproteinuric effects which act directly by protecting slit diaphragm molecules, including nephrin and podocin $[8,9]$, while cisplatin-induced kidney damage is considered to be mediated by angiotensin-II [10, 11]. In fact, the protective effects of ARBs on testis have been previously reported [12-14].

Taken together, in this study, we initially examined the effects of cisplatin-induced damage on expression patterns of 
nephrin and podocin in rat testes, since platinum analogues like cisplatin are known to have toxic side effects on testicular function and frequently cause azoospermia through the impaired function of Sertoli cells and disruption of blood/ testis barrier [15-18]. We then evaluated whether this damage could be restored by the angiotensin-II receptor blocker (ARB), candesartan.

\section{Materials and Methods}

2.1. Animals. A total of forty-two 9-week-old male SpragueDawley rats weighting $234 \pm 23 \mathrm{~g}$ were purchased from Oriental Yeast Co. (Tokyo, Japan) and housed in a controlled environment at $22^{\circ} \mathrm{C}$ on a 12-hour light, 12-hour dark cycle. Animal experiments were approved by Committee of Animal Experiment at Kobe University School of Medicine and conducted in accordance with the National Institutes of Health Guide for the Care and Use of Laboratory Animals [19].

2.2. Sertoli Cell Line. The mouse Sertoli cell line TM4 was purchased from the American Type Culture Collection (Manassas, Virginia). Cells were maintained in Dulbecco's Modified Eagle Medium/F12 (DMEM/F12) (Nacalai Tesque, Kyoto, Japan), supplemented with $10 \%$ fetal bovine serum. This cell line TM4 is known to form tight junction and is considered suitable for this study [20].

2.3. Animal Studies. To examine the in vivo effects of treatment with cisplatin (Nichi-Iko Pharmaceutical Co., Toyama, Japan) and/or candesartan, kindly provided by Takeda Pharmaceutical Co. (Osaka, Japan), on expression patterns of testicular nephrin and podocin, a total of 42 rats mentioned above were randomly selected for the following six groups: Group $1(n=7)$, intraperitoneal (IP) injection of $2 \mathrm{~mL}$ isotonic saline; Group $2(n=7)$, IP injection of $2 \mathrm{~mL}$ isotonic saline and oral administration of candesartan $5 \mathrm{mg} / \mathrm{kg} /$ day; Group $3(n=7)$, IP injection of $4 \mathrm{mg} / \mathrm{kg}$ cisplatin; Group 4 $(n=7)$, IP injection of $4 \mathrm{mg} / \mathrm{kg}$ cisplatin and oral administration of candesartan $5 \mathrm{mg} / \mathrm{kg} /$ day; Group $5(n=7)$, IP injection of $7 \mathrm{mg} / \mathrm{kg}$ cisplatin; and Group $6(n=7)$, IP injection of $7 \mathrm{mg} / \mathrm{kg}$ cisplatin and oral administration of candesartan $5 \mathrm{mg} / \mathrm{kg} /$ day. After randomization, either candesartan at a dose of $5 \mathrm{mg} / \mathrm{kg}$ or water was orally administered once daily for 24 days, and on day 3 , either cisplatin at a dose of 4 or $7 \mathrm{mg} / \mathrm{kg}$ or isotonic saline was given by IP injection. The dose and duration after treatment of cisplatin and candesartan were determined based on those reported in the previous study and our preliminary experiments [21, 22].

Three weeks after treatment, rats were sacrificed, and testes and epididymides were removed. Testicular weight measurement and epididymal semen analysis were performed on both sides, and mean values were used for the calculation. After semen analysis, testes were bisected, and one half was snap-frozen immediately and stored at $-80^{\circ} \mathrm{C}$ until assessed, and the remainder was fixed in Bouin solution for histopathological examination.
2.4. Sperm Analysis. The epididymal sperm concentration was determined by using the modified method described by Okada et al. [23]. Briefly, the epididymis was finely minced by anatomical scissors within $1 \mathrm{~mL}$ of modified Whitten's medium (15 mM Hepes-sodium salt, $1.2 \mathrm{mM} \mathrm{MgCl}$, $100 \mathrm{mM}$ $\mathrm{NaCl}, 4.7 \mathrm{mM} \mathrm{KCl}, 1 \mathrm{mM}$ pyruvic acid, and $4.8 \mathrm{mM}$ lactic acid) in a dish and then allowed to incubate at room temperature for 1 hour to provide the migration of all spermatozoa from epididymis. Sperm analysis, including the number and motility, was performed using Makler counting chamber (Irvine Scientific, CA, USA). A total of 20 fields were analyzed for each sperm sample. In order to detect the spermatozoa with abnormal morphologies, the Diff-Quick kit (Medion Diagnostics International, FL, USA) was used to stain the smears of semen samples. Diff-Quick fixing and staining times were 5 minutes and the slides were washed in distilled water to eliminate excess staining, air-dried, and covered with a coverslip. At least 100 sperm per slide were counted to determine the percentage of spermatozoa with abnormal morphologies.

2.5. Treatment of TM4 Cells. To examine the changes in expression patterns of nephrin and podocin in the cultured TM4 cells, the cells were treated with $2 \times 10^{-8} \mathrm{~mL} / \mathrm{L}$ angiotensin-II (A9525, Sigma-Aldrich, Tokyo, Japan) for 1 hour in the presence or absence of $2.5 \times 10^{-6} \mathrm{~mol} / \mathrm{L}$ candesartan. Following treatment, cells were harvested and used for subsequent experiments. Angiotensin-II is considered suitable for our experiment, to investigate the involvement of angiotensin system through nephrin and podocin. That is, angiotensin receptor is located in Sertoli cell, while there is no production of angiotensin-II from Sertoli cell [24, 25].

2.6. Real-Time RT-PCR. Total ribonucleic acid (RNA) was extracted from the rat testes and cultured TM4 cells using TRIzol reagent (Life Technologies Japan, Tokyo, Japan). RT reactions were carried out using a GeneAmp RNA PCR Kit (Applied Biosystems, Foster City, California) according to the manufacturer's instructions. To quantitatively determine the expression levels of nephrin, podocin, and GAPDH mRNAs in each sample, real-time PCR analysis using a standard curve method with SYBR Green I (Takara Bio, Tokyo, Japan) was then conducted as previously described [26]. The sequencespecific primers, synthesized by Operon Biotechnology Inc. (Tokyo, Japan) based on previous studies, are presented in Table 1 [27, 28]. Data were normalized with glyceraldehyde 3 -phosphate dehydrogenase (GAPDH) values and expressed as arbitrary units relative to samples from control, regarded as "1."

2.7. Western Blot Analysis. Samples containing equal amounts of protein $(20 \mu \mathrm{g})$ from lysates of the rat testes or cultured TM4 cells were subjected to SDS-polyacrylamide gel electrophoresis and transferred to a nitrocellulose filter. The filter was blocked in PBS containing 5\% nonfat milk powder at $4^{\circ} \mathrm{C}$ overnight and then incubated for 1 hour with antibodies against nephrin $(1: 200$, sc-32532, Santa Cruz 
TABle 1: The species-specific primers of podocin, nephrin and GAPDH.

\begin{tabular}{lll}
\hline & Sense primer & Antisense primer \\
\hline Rat & & \\
Podocin & CCTGTGAGTGGCTTCTTGTCCTC & GGAGACGCTTCATAGTGGTTTGCA \\
Nephrin & CTGACTGGGCTGAAGCCTTCT & AAGAGCACAGGCAGCAGGGG \\
GAPDH & CTCTACCCACGCCAAGTTCAA & GGATGACCTTGCCCACAGC \\
Mouse & & \\
Podocin & TGAGGATGGCGGCTGAGAT & GGTTTGGAGGAACTTGGGT \\
Nephrin & CCCAACACTGGAAGAGGTGT & CTGGTCGTAGATTCCCCTTG \\
GAPDH & CTCATGACCACAGTCCATGC & CACATTGGGGGTAGGAACAC \\
\hline
\end{tabular}

Biotechnology, Santa Cruz, California), podocin (1:200, P0372, Sigma-Aldrich, Tokyo, Japan), and $\beta$-actin $(1: 1000$, A1978, Sigma-Aldrich). The filters were then incubated for 30 minutes with horseradish peroxide-conjugated secondary antibodies $(1: 2000$, sc-2004, sc-2020 Santa Cruz Biotechnology), and specific proteins were detected using an enhanced chemiluminescence Western blot analysis system (RPN2106, GE Healthcare, Tokyo, Japan). The relative optical densities of the bands were quantified using Image J Program [29], and data were expressed as corresponding values of the ratio relative to the result from control group.

2.8. Immunohistochemical Studies. Immunofluorescence staining was used to investigate the expression of nephrin and podocin. For frozen sections, antibodies against nephrin and podocin as described above were added in dilution of $1: 200$ and 1:400, respectively, and then incubated for 2 hours. After staining with the secondary antibodies goat antirabbit IgG-TR (1:500, sc-2780, Santa Cruz Biotechnology) and donkey anti-goat IgG-FITC (1:500, sc-2024, Santa Cruz Biotechnology), the sections were mounted and the staining outcomes were evaluated by inverted fluorescence phasecontrast microscopy (Keyence BZ-9000, Osaka, Japan). For in vitro studies, cultured TM4 cells were seeded on coverslips and fixed in $4 \%$ paraformaldehyde and were stained with primary and secondary antibodies, the same as in vivo study, and counterstaining with $4^{\prime}, 6$-diamidino-2-phenylindole (DAPI) was additionally performed.

2.9. TUNEL Staining. Apoptotic features in the rat testes were evaluated by Terminal Deoxynucleotidyl Transferasemediated dUTP Nick End-labelling (TUNEL) assay using an in situ cell death detection kit (11684795910, Roche Applied Science, Penzberg, Germany) according to the manufacturer's instructions. Quantitative analysis was performed by counting the number of apoptotic cells and total germ cells in 100 randomly selected tubules in one slide per animal.

2.10. Immunofluorescence Staining of Carbonylated Protein. Carbonylated protein is known as a marker of oxidative stress, since protein carbonylation has been identified as a potential mechanism underlying mitochondrial dysfunction which is linked to oxidative stress $[30,31]$. The expression of carbonylated protein in the rat testes was evaluated using a protein carbonyls immunohistochemical staining kit (Cosmo Bio, Tokyo, Japan). In accordance with the manufacturer's protocol, methacarn-fixed paraffin-embedded sections were incubated with anti-dinitrophenylhydrazine (DNP) antibody, followed by incubation with fluorescein isothiocyanate (FITC) conjugated secondary antibody for 1 hour at room temperature in the dark. After the labelling procedure was completed, the coverslips were mounted onto glass slides using a mounting medium containing $4^{\prime}, 6$-diamidino-2phenylindole.

2.11. Superoxide Dismutase (SOD) Assay. To quantify the production of superoxide in the rat testes, Superoxide Dismutase (SOD) activity was measured using an SOD Assay Kit-WST (DOJINDO, Kumamoto, Japan) based on the manufacturer's protocol. In this assay, homogenized tissue samples were centrifuged and the supernatant was processed for the measurement of SOD activity as previously described [32]. One unit of SOD activity was defined as the amount of enzyme that inhibits $50 \%$ of the WST-1 formazan per minute.

2.12. Immunoprecipitation. Immunoprecipitation analysis was performed as previously described procedure [33]. Homogenized samples were precleared by incubation with protein G-plus-agarose beads (sc-2003, Santa Cruz Biotechnology) at $4^{\circ} \mathrm{C}$ for 1 hour and then incubated with primary antibodies as described above. Agarose beads were washed with lysis buffer and suspended in SDS/PAGE sample buffer. After SDS-polyacrylamide gel electrophoresis, samples were analyzed by Western blotting with corresponding antibodies.

2.13. Statistical Analysis. The significance of differences was evaluated by the Mann-Whitney $U$ test for unpaired observations. Values were expressed as the mean \pm standard deviation, and $P$ values $<0.05$ were considered significant.

\section{Results}

The changes in testicular weight and sperm analysis following treatment with cisplatin and/or candesartan are summarized in Figure 1. Cisplatin caused the dose-dependent testicular damage; administration of high-dose $(7 \mathrm{mg})$ cisplatin caused a significant decrease in testicular weights, while low-dose (4 mg) cisplatin did not affect the testicular weight. These 

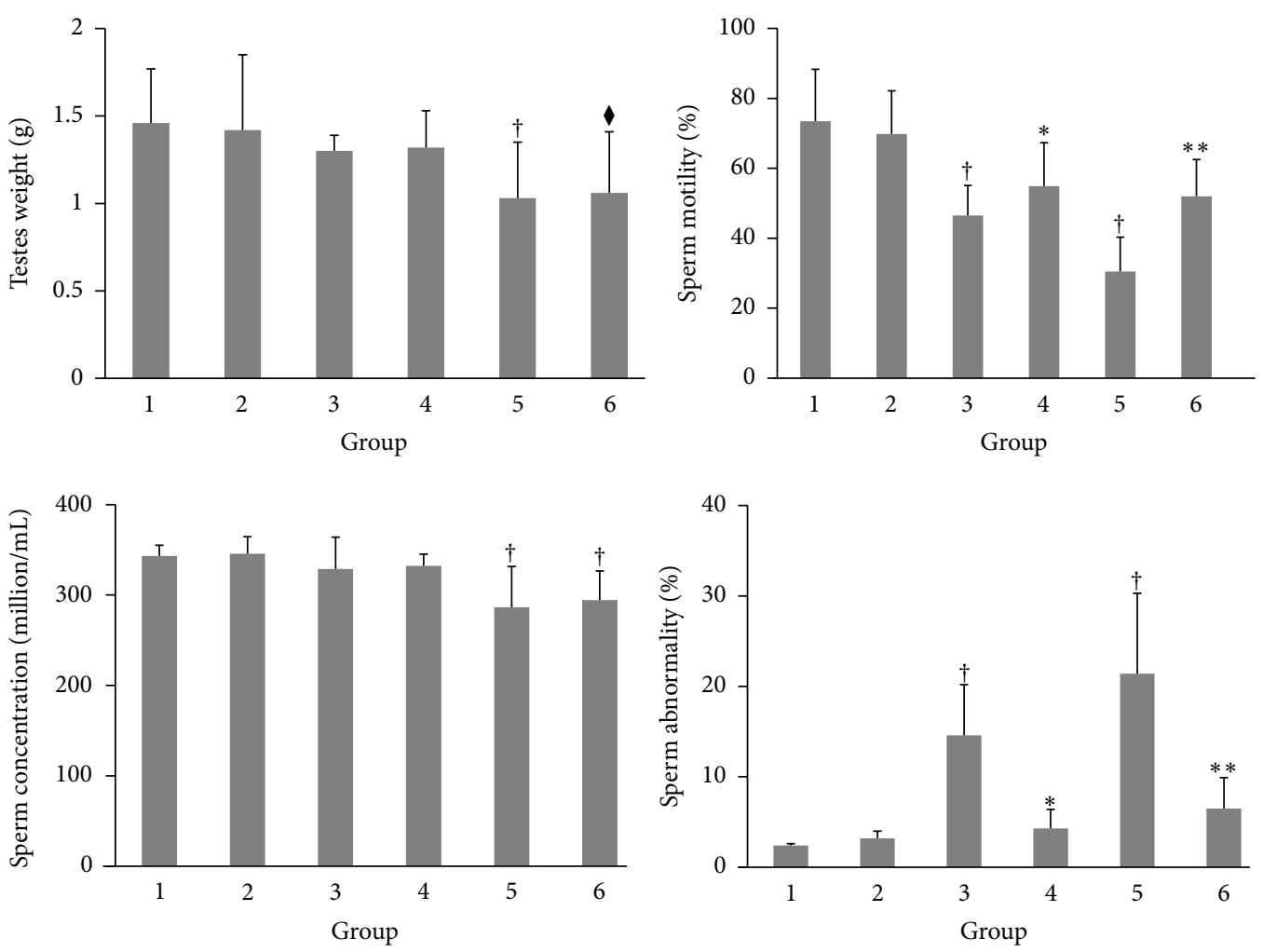

FIGURE 1: Weights of testes, epididymal sperm concentrations, and percentages of sperm motilities and abnormalities following treatment with cisplatin and/or candesartan. Group $1(n=7)$, intraperitoneal (IP) injection of $2 \mathrm{~mL}$ isotonic saline; Group $2(n=7)$, IP injection of $2 \mathrm{~mL}$ isotonic saline and oral administration of candesartan $5 \mathrm{mg} / \mathrm{kg} /$ day; Group $3(n=7)$, IP injection of $4 \mathrm{mg} / \mathrm{kg}$ cisplatin; Group $4(n=7)$, IP injection of $4 \mathrm{mg} / \mathrm{kg}$ cisplatin and oral administration of candesartan $5 \mathrm{mg} / \mathrm{kg} /$ day; Group $5(n=7)$, IP injection of $7 \mathrm{mg} / \mathrm{kg}$ cisplatin; and Group $6(n=7)$, IP injection of $7 \mathrm{mg} / \mathrm{kg}$ cisplatin and oral administration of candesartan $5 \mathrm{mg} / \mathrm{kg} / \mathrm{day}$. Each column represents the mean value with standard deviation. ${ }^{\dagger},{ }^{*}$, and ${ }^{* *}$ differ from Groups 1,3 , and 5 , respectively $(P<0.05)$.

changes were not affected by candesartan treatment. Sperm analysis revealed that sperm concentration and motility were significantly decreased by cisplatin administration, and increased proportion of abnormal sperm was observed after cisplatin administration. Coadministration of candesartan significantly recovered the motility rate and sperm abnormality rate. Sperm concentration was slightly recovered by candesartan, but the difference was not statistically significant.

We then assessed the changes in the expression levels of nephrin and podocin in the rat testes following treatment with cisplatin and/or candesartan. The expression of both nephrin and podocin mRNAs was detectable in the rat testes, and these levels were significantly decreased by treatment with cisplatin (Figures 2(a) and 2(b)); however, these changes were partially, but significantly, restored by combined administration with candesartan. Changes in the protein expression levels of both nephrin and podocin after treatment with cisplatin and/or candesartan were similar to those in the nephrin and podocin mRNAs (Figures 2(c)-2(e)).

Expression patterns of nephrin and podocin in the rat testes following treatment with cisplatin and/or candesartan are presented in Figure 3. Dual-labeling immunofluorescence staining revealed colocalization of nephrin and podocin near the basal membrane of seminiferous tubules. Moreover, the administration of cisplatin markedly reduced the staining intensities of both nephrin and podocin and resulted in a shift of both proteins from linear to granular expression patterns, accompanying the dissociation of these two molecules. These changes induced by cisplatin were partially restored by combined treatment with candesartan.

The effects of treatment with cisplatin and/or candesartan on apoptotic features in the rat testes were subsequently investigated. As shown in Figure 4, a marked increase in the number of TUNEL-positive cells was observed in the group treated with cisplatin in a dose-dependent manner, while the number of TUNEL-positive cells was significantly reduced in the group receiving candesartan in addition to cisplatin. Furthermore, the assessment of oxidative stress revealed that treatment with cisplatin induced a marked increase in the expression of carbonylated protein in the rat testes compared with the baseline expression in the control group without cisplatin treatment, and coadministration of candesartan partially inhibited the cisplatin-induced upregulation of this protein (Figures 5(a) and 5(b)). Similarly, a quantitative assessment of SOD activity in the rat testes showed a cisplatin-induced decrease in this activity and its partial restoration by candesartan (Figure 5(c)).

To confirm the findings of in vivo experiments, in vitro studies using a cultured mouse Sertoli cell line, TM4, were conducted. In TM4 cells as well, nephrin and podocin were 

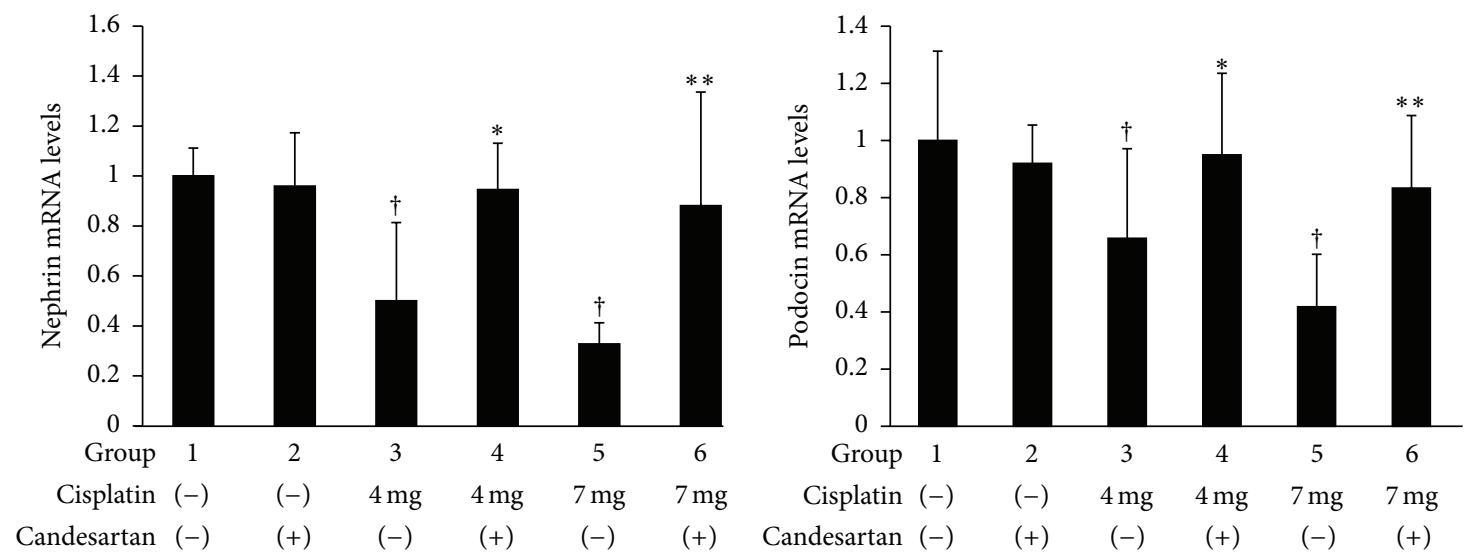

(a)

(b)

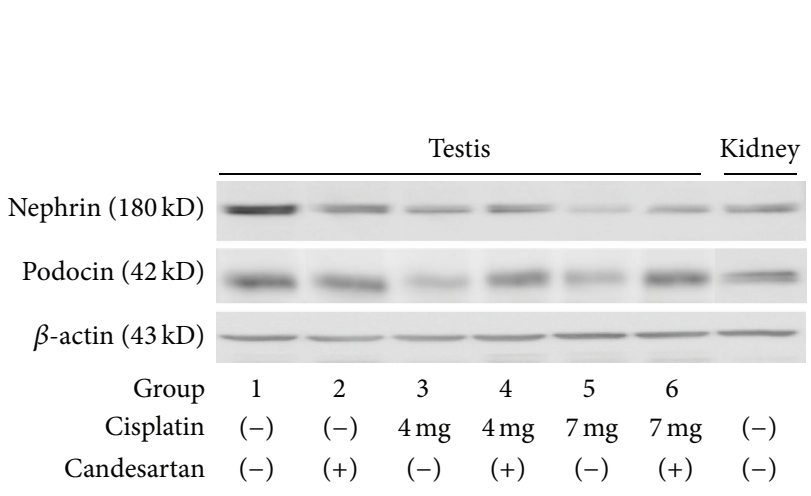

(c)

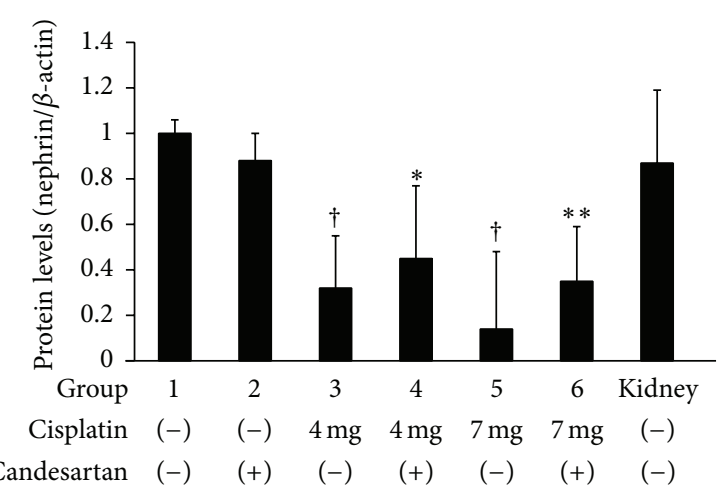

(d)

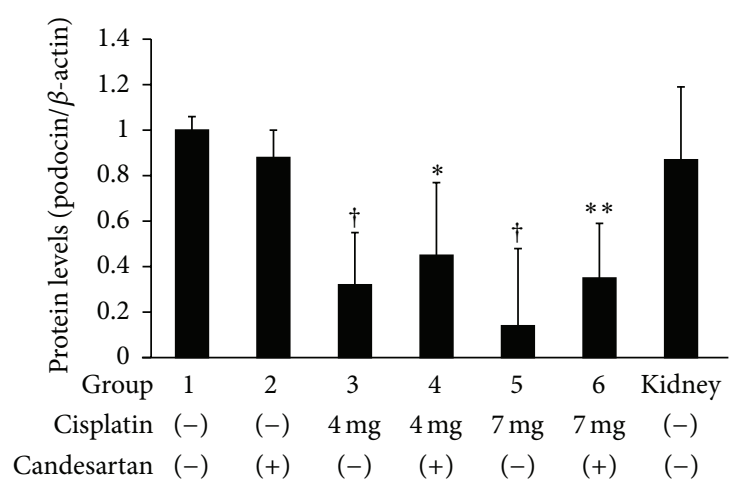

(e)

FIGURE 2: Changes in expression levels of nephrin and podocin in the testes of rats following treatment with cisplatin and/or candesartan. (a) Total RNA was extracted from the testes and analyzed for nephrin mRNA levels by real-time RT-PCR. Each column represents the mean value with standard deviation. ${ }^{\dagger},{ }^{*}$, and ${ }^{* *}$ differ from Group 1 , Group 3, and Group 5, respectively $(P<0.05)$. (b) Total RNA was extracted from the testes and analyzed for podocin mRNA levels by real-time RT-PCR. Each column represents the mean value with standard deviation. $\dagger,{ }^{*}$, and ${ }^{* *}$ are represented as in (a). (c) Protein was extracted from the testes, and expression levels of nephrin, podocin, and $\beta$-actin were analyzed by Western blotting. (d) Nephrin and $\beta$-actin signal intensity in (c) were quantified, and the ratio of nephrin over $\beta$-actin (d) and that of podocin over $\beta$-actin (e) were presented in histogram format. ${ }^{\dagger},{ }^{*}$, and ${ }^{* *}$ are represented as in (a).

definitely expressed at both mRNA and protein levels, and treatment with angiotensin-II induced a reduction of their expression levels, which could be partially recovered by additional treatment with candesartan (Figure 6). As shown in Figure 7(a), immunofluorescence staining revealed that the expression of nephrin and podocin was mainly localized at junctions between TM4 cells cultured in standard medium; however, exposure of TM4 cells to angiotensin-II induced redistribution of these proteins, resulting in the emergence of diffused localization patterns. These changes in expression patterns of both proteins could be restored by combined treatment with candesartan. 

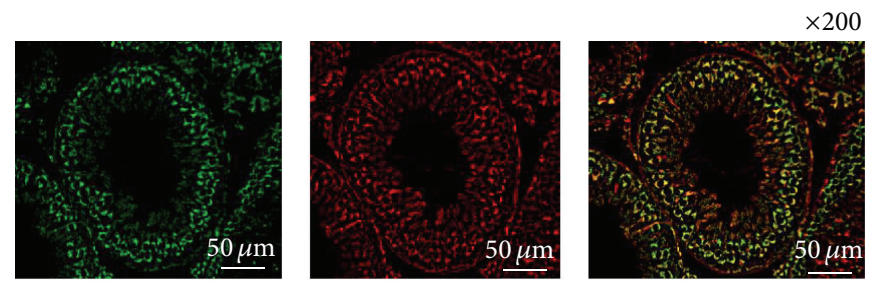

Group 1: control
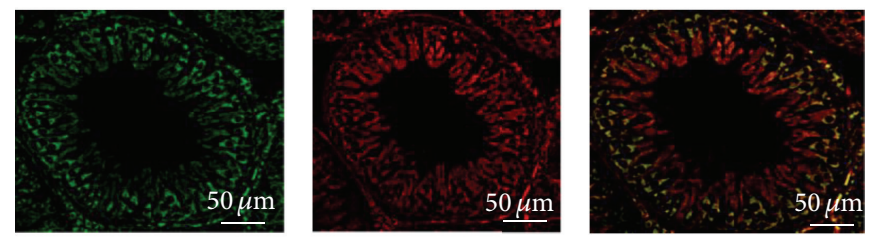

Group 2: candesartan
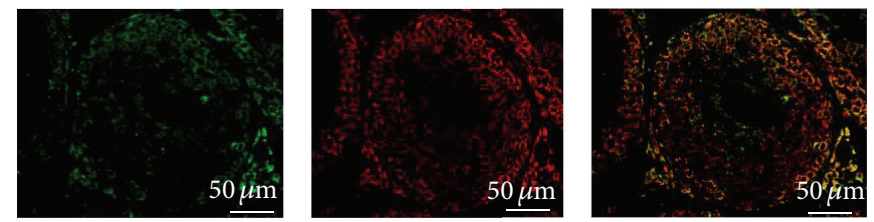

Group 3: cisplatin (4 mg)
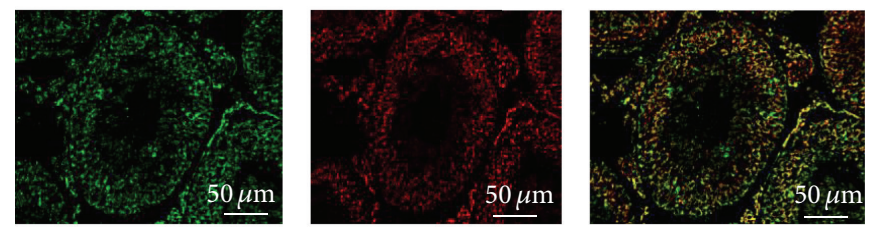

Group 4: cisplatin (4 mg)

+ candesartan
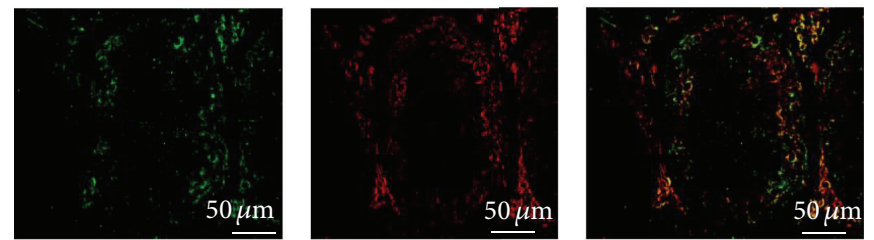

Group 5: cisplatin (7 mg)
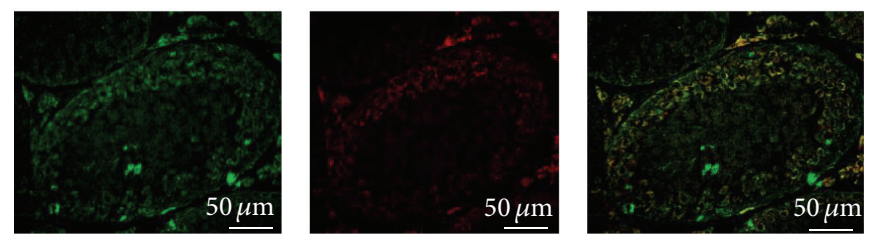

Group 6: cisplatin (7 mg)

+ candesartan

Nephrin

Podocin

Overlay (nephrin + podocin)

(a)
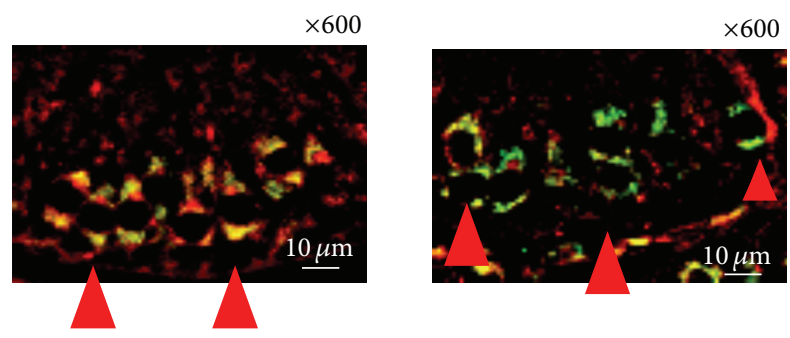

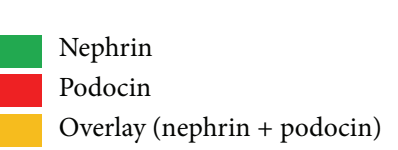

(b)

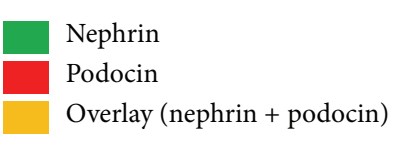

(c)

Figure 3: Continued. 


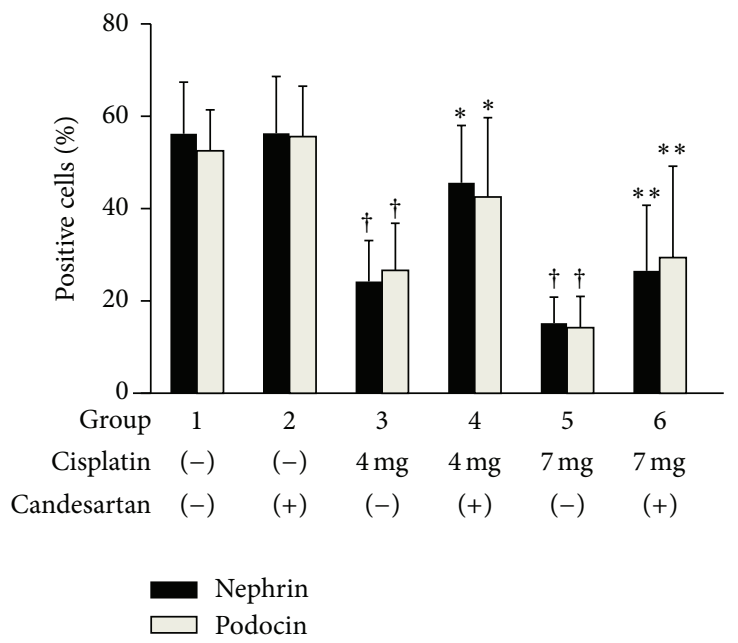

(d)

FIGURE 3: Immunofluorescence double staining for nephrin and podocin in the testes of rats following treatment with cisplatin and/or candesartan. Nephrin was stained in green with FITC, podocin was stained in red with Texas Red, and the nuclei were stained in blue with DAPI. The overlaps of nephrin and podocin are indicated in orange. (a) Representative findings of immunofluorescence double staining and changes in each group. (b) High magnification images of control testis. Arrows indicate that nephrin and podocin are coexpressed in linear formation near basal membrane of seminiferous tubules. (c) High magnification images of testis following treatment with cisplatin. Arrows indicate that, after cisplatin $(7 \mathrm{mg})$ administration, the expression patterns of nephrin and podocin were dissociated and in discrete granular structure. (d) Quantitative analysis of immunofluorescence positive cells for nephrin and podocin. ${ }^{\dagger},{ }^{*}$, and ${ }^{* *}$ differ from Group 1, Group 3, and Group 5, respectively $(P<0.05)$.

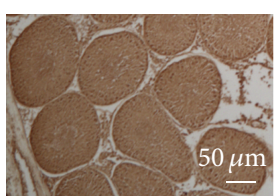

Group 1: control

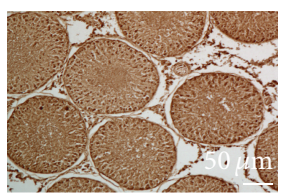

Group 4: cisplatin (4 mg) + candesartan

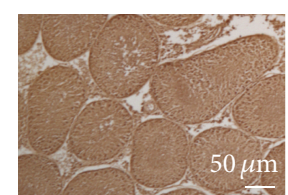

Group 2: candesartan

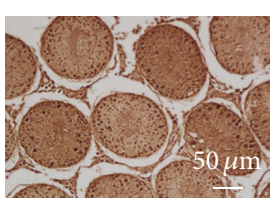

Group 5: cisplatin (7 mg)

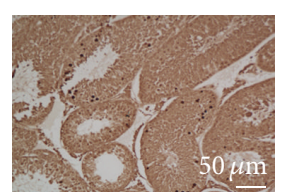

Group 3: cisplatin (4 mg)

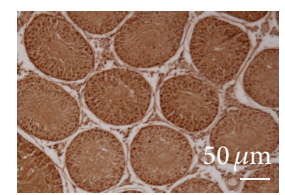

Group 6: cisplatin (7 mg) + candesartan (a)

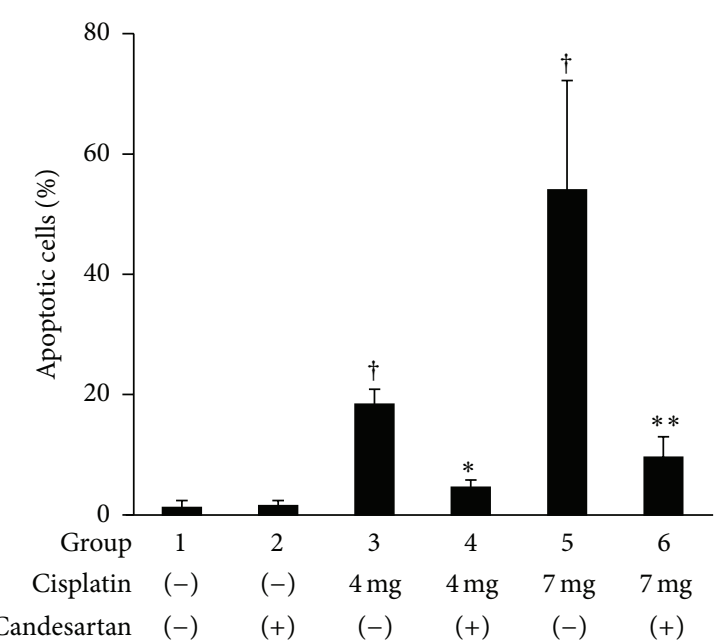

(b)

FIgURE 4: Apoptotic features in the testes of rats following treatment with cisplatin and/or candesartan. (a) Representative findings of TUNEL staining in rat testes. (b) The numbers of TUNEL-positive cells and total germ cells in 100 randomly selected tubules in one slide per animal were counted. Apoptotic index, defined as the percentage of TUNEL-positive cells, was then calculated for each slide. Each column represents the mean value with standard deviation. ${ }^{\dagger},{ }^{*}$, and ${ }^{* *}$ differ from Group 1, Group 3, and Group 5, respectively $(P<0.05)$.

To examine whether nephrin specifically binds to podocin in TM4 cells, immunoprecipitation assay was performed. As shown in Figure 7(b), nephrin was coimmunoprecipitated with podocin, while podocin was also coimmunoprecipitated with nephrin.

\section{Discussion}

During the last two decades, several molecules have been identified to be associated with the structure of the slit diaphragm in the kidney podocyte, the primary filtration 


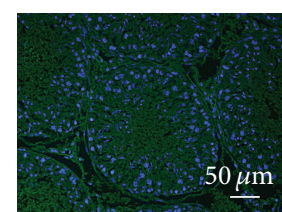

Group 1: control

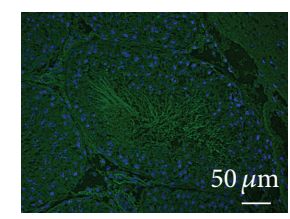

Group 4: cisplatin (4mg) + candesartan

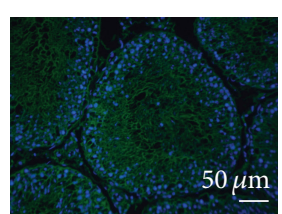

Group 2: candesartan

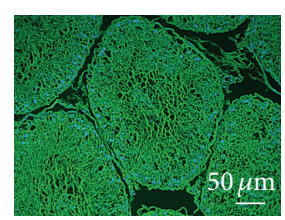

Group 5: cisplatin $(7 \mathrm{mg})$

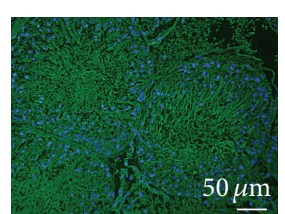

Group 3: cisplatin (4 mg)

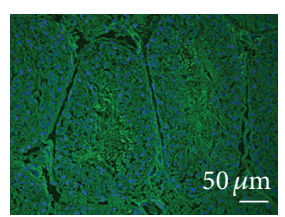

Group 6: cisplatin (7 mg) + candesartan

(a)

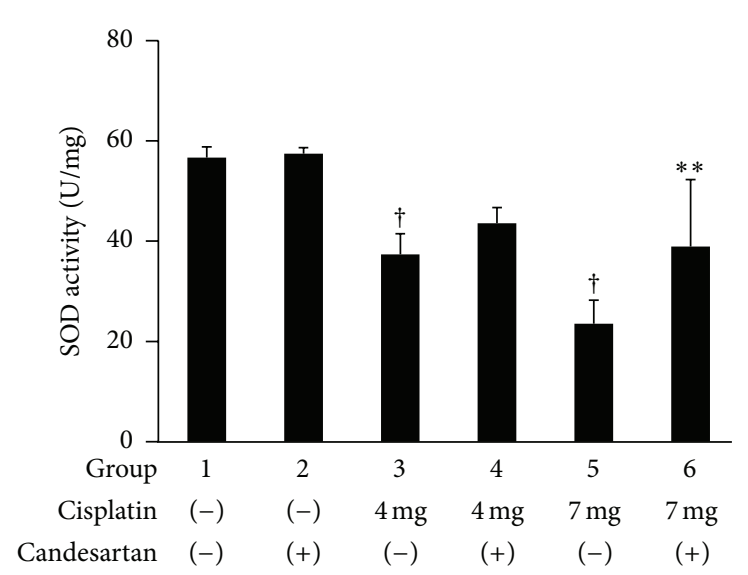

(c)

Figure 5: Oxidative stress status in the testes from rats following treatment with cisplatin and/or candesartan. (a) Representative findings on immunofluorescent staining of carbonylated protein in the rat testes. (b) Carbonylated protein signal intensities in (a) were quantified by Image J software and the ratio of each group over Group 1 was presented in histogram format. (c) Homogenized testicular specimens were prepared and analyzed for SOD activity. Each column represents the mean value with standard deviation. ${ }^{\dagger},{ }^{*}$, and ${ }^{* *}$ differ from Group 1 , Group 3, and Group 5, respectively $(P<0.05)$.

barrier responsible for macromolecule proteins. The initially reported molecule consisting of the slit diaphragm was ZO-1, originally identified as a component of the tight junction [34]. Nephrin and podocin, also well characterized components of the slit diaphragm, were shown to bind to each other, and several studies suggested that abnormal or insufficient signaling via the nephrin-podocin complex is likely to result in the development of proteinuria $[8,27,35]$. Recently, Relle et al. reported that the expression of podocin was detected in the testes and that confocal laser microscopy revealed colocalization of podocin with filamentous actin within Sertoli cells, suggesting a possible role of podocin in the blood-testis barrier [6]. Liu et al. reported the testicular expression of nephrin as well, which was colocalized with ZO-1 [7]. In this study, we revealed the colocalization of nephrin and podocin near the basal membrane of seminiferous tubules. These localization patterns were similar to those of tight junction protein, such as $\mathrm{F}$-actin, $\beta$-catenin, and $\mathrm{N}$-cadherin $[6,36]$. Collectively, these findings indicate that nephrin and podocin may be involved in the function mediating the barrier system in cell-cell junction in the testis.

In this study, in order to investigate the relationship between testicular damage and expression patterns of nephrin and podocin in the testes, we treated rats with cisplatin, which has been shown to have a potential toxic effect on the testes through the impairment of functions associated with cell-cell adhesion $[17,37]$. We initially confirmed a significant decrease in sperm concentration and motility as well as increased proportion of abnormal sperm in rats treated with cisplatin. Furthermore, cisplatin treatment induced marked inhibitory effects on the expression of testicular nephrin and podocin. Immunohistochemical analyses also showed that nephrin and podocin were colocalized near the basal membrane of seminiferous tubules without cisplatin treatment; however, the dissociated expression of these proteins, accompanying the changes in granular expression patterns, was observed following the administration of cisplatin. These findings suggest the possible role of nephrin and 


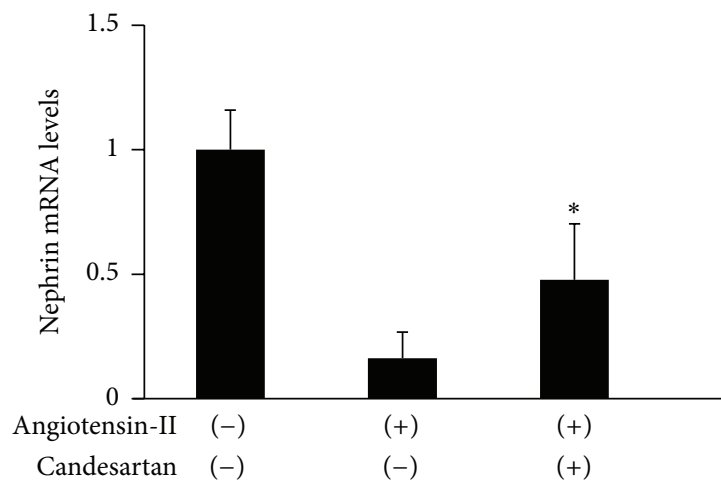

(a)

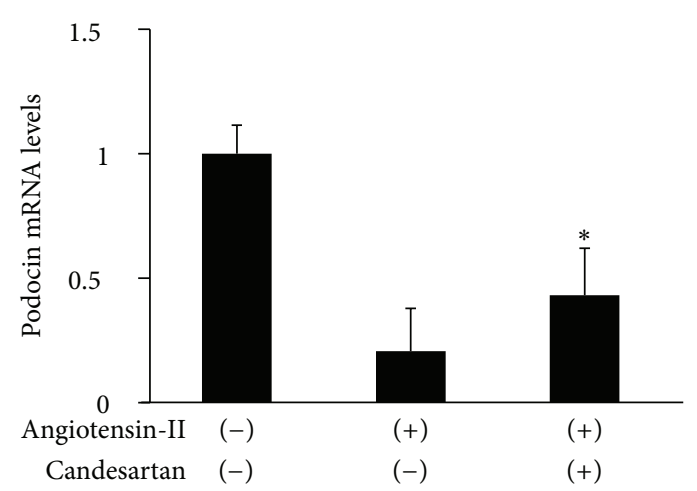

(b)

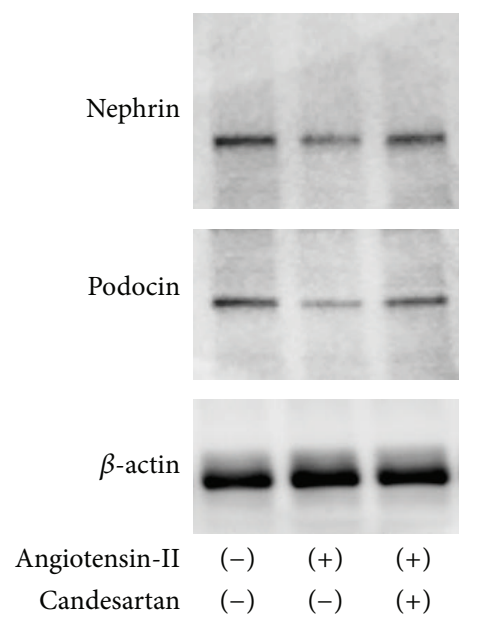

(c)

FIgURE 6: Changes in expression levels of nephrin and podocin in cultured mouse Sertoli cells, line TM4, following treatment with angiotensin-II and/or candesartan. (a) Total RNA was extracted from TM4 cells and analyzed for nephrin mRNA levels by real-time RTPCR. Each column represents the mean value with standard deviation. ${ }^{*}$ differs from TM4 cells treated with cisplatin alone $(P<0.05)$. (b) Total RNA was extracted from the testes and analyzed for podocin mRNA levels by real-time RT-PCR. Each column represents the mean value with standard deviation. ${ }^{*}$ differs from TM4 cells treated with angiotensin-II alone $(P<0.05)$. (c) Protein was extracted from TM4 cells and expression levels of nephrin, podocin, and $\beta$-actin were analyzed by Western blotting.

podocin in cisplatin-induced damage through the disruption of the barrier function in the testes.

It is of interest to investigate whether cisplatin-induced testicular damage can be alleviated by an antiproteinuric agent. It has been well recognized that ARBs help reduce renal tissue damage and proteinuria in some renal diseases through specific blockade of angiotensin-II type 1 receptor (AT1R), independently of its function to lower blood pressure [32, 33]. Furthermore, several recent studies have demonstrated that AT1R-mediated action directly protects slit diaphragm molecules, including nephrin and podocin, from stimuliinducing renal injury $[8,9,38,39]$. To evaluate the regulatory mechanism of nephrin and podocin by angiotensinII in addition to these findings, we assessed the effects of candesartan, one of the most prevalent ARBs, on the damage in rat testes following cisplatin treatment. Our results showed that the administration of candesartan resulted in the improvement of cisplatin-induced toxic events in the rat testis, including decreased sperm motility, and increased proportion of abnormal sperm. In addition, the reduced expression of nephrin and podocin and their dissociated expression patterns accompanied by apoptosis were partially restored by candesartan administration. Other than the present findings, there have been several studies revealing the involvement of an angiotensin-mediated system in the maintenance of testicular function [12-14, 40, 41]. For example, Shiraishi et al. reported that AT1R-dependent fibrosis in rat testes was observed after vasectomy and that impaired spermatogenesis caused by interstitial fibrosis was partially abrogated by ARB administration [14]. Similarly, Kushwaha and Jena reported that testicular toxicity induced by nicotine in streptozotocininduced diabetic rat was attenuated by ARB treatment [13]. Taken together, these findings indicate that ARB may have protective effects from testicular damage induced by several types of toxic stimuli to the testes. 

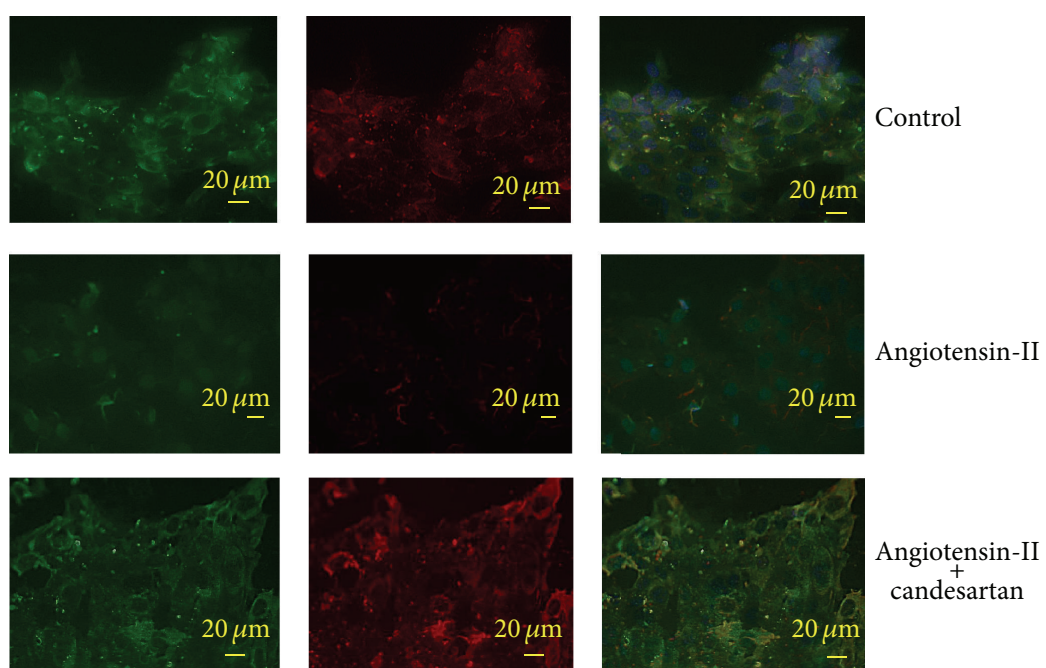

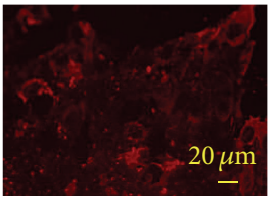

Podocin

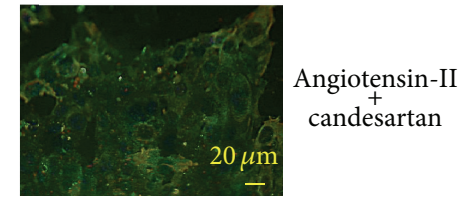

Overlay
Nephrin

(a)

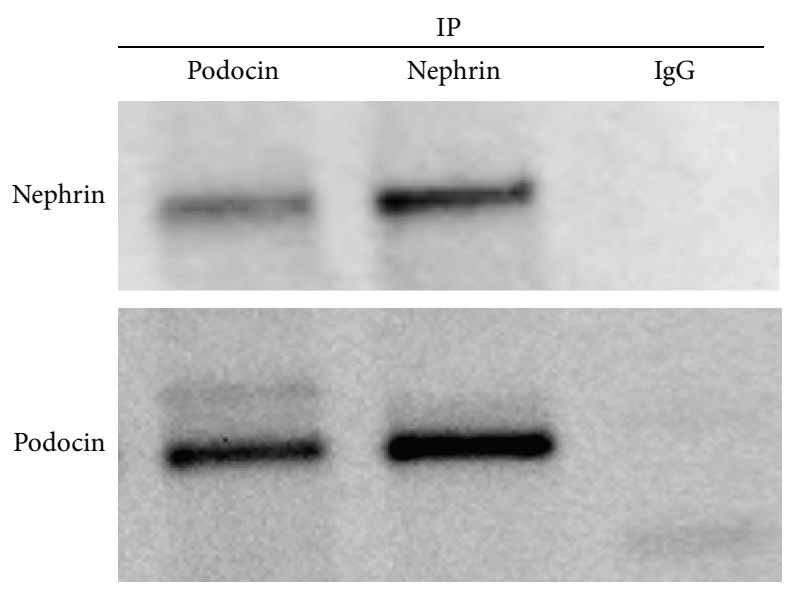

(b)

FIGURE 7: (a) Immunofluorescence double staining for nephrin and podocin in cultured mouse Sertoli cells, line TM4, following treatment with angiotensin-II and/or candesartan. Nephrin was stained in green with FITC, podocin was stained in red with Texas Red, and the nuclei were stained in blue with DAPI. The overlaps of nephrin and podocin are indicated in orange. Nephrin and podocin are mainly located at junctions between cells, and administration of angiotensin-II induced redistribution of nephrin and podocin. These changes were partially restored by additional treatment with candesartan. (b) Coimmunoprecipitation (IP) assays of nephrin and podocin in cultured mouse Sertoli cells, line TM4. Whole-cell lysates prepared from TM4 cells were immunoprecipitated with antipodocin or nephrin antibody and blotted with antinephrin or podocin antibody, respectively.

Another point of interest is the mechanism mediating the protective effects on cisplatin-induced testicular damage by candesartan. To date, a number of previous studies have reported the protective effects of ARB on testicular damage. Some of these reports clarified the antioxidative activity induced by $\mathrm{ARB}$ as one of the main mechanisms underlying its protective effect on testicular damage $[13,14]$. In fact, there have been several studies showing the induction of reactive oxygen species (ROS) through AT1R as well as the antioxidant activity of ARB in various types of cells [42] (Figure 8). Griendling et al. reported that angiotensin-II specifically activates enzyme systems that promote superoxide generation in cultured vascular smooth muscle cells [43]. Similarly, Okui et al. showed that cisplatin increased serum levels of angiotensin-II and aldosterone, which synergistically prolonged the accumulation of cisplatin in renal tissues [10]. In the testes as well, ROS has been shown to produce detrimental effects on the maintenance of their physiological functions [44]. Furthermore, antioxidants have been regarded as having protective effects on testicular damage [45-47]. For example, Salem et al. also reported the attenuation of cisplatin-induced testicular toxicity by antioxidant supplementation with selenium and lycopene [48]. Similarly to these studies, we also confirmed the restoring effect of candesartan on increased oxidative stress induced by cisplatin in rat testes. Considering these findings, candesartan may alleviate cisplatin-induced testicular damage through the inhibition of oxidant activity. However, in addition to these 


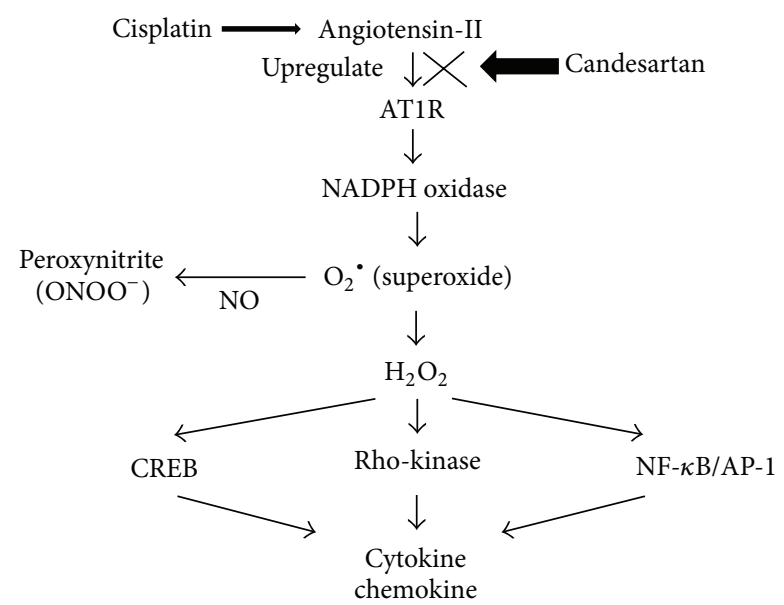

FIGURE 8: Signaling pathway of angiotensin-II type 1 leading to superoxide production. AT1R: angiotensin-II type 1 receptor, NADPH: nicotinamide adenine dinucleotide, CREB: cAMP response element binding protein, and NO: nitric oxide.

findings, we have to mention the toxic effects of cisplatin on cells in the adluminal compartment, which are normally protected by the blood/testis barrier, indicating that cisplatin and candesartan might modulate the functions of blood/testis barrier. In particular, ARB mediated protective effects on cellcell junction in the testes, including nephrin and podocin. In this study, we confirmed that the dissociated nephrinpodocin complex was recovered by ARB administration, indicating that $A R B$ could have protective effects among blood/testis barrier architecture.

In order to confirm and reinforce the findings obtained from in vivo studies, we also performed several in vitro experiments using a mouse Sertoli cell line, TM4. As for the changes in expression patterns of nephrin and podocin in TM4 cells following treatment with angiotensin-II and/or candesartan, we achieved findings that angiotensin-II directly acts on Sertoli cells and affects the expression of nephrin and podocin. Furthermore, the immunoprecipitation assay demonstrated binding between nephrin and podocin in TM4 cells. To our knowledge, this is the first study providing evidence concerning the binding of these proteins in cells other than those derived from the kidney. Although additional studies will be necessary to address the functional significance of the binding between testicular nephrin and podocin, these findings strongly suggest a direct interaction between these proteins in the testes like that in the renal slit diaphragm.

Here, we would like to emphasize several limitations of this study. Firstly, treatment of cisplatin was selected to induce damage in the testes; therefore, it is impossible to apply the findings of this study to all types of testicular injury. Secondly, we did not directly address whether testicular nephrin and podocin colocalized in tight junction in seminiferous tubules and regulate the physiological testicular functions or bloodtestis barrier. Thus, it would be required to perform further study, such as that using immunoelectron microscopy and that assessing blood-testis barrier permeability.
Thirdly, the significance of the use of candesartan together with chemotherapeutic agents from a clinical viewpoint should be evaluated. Finally, expression patterns of the nephrin-podocin complex in clinical testicular specimens from infertile men should be investigated to assess the role of these proteins in spermatogenesis.

\section{Conclusion}

Candesartan can prevent cisplatin-induced testicular damage by regulating expression patterns of the nephrin-podocin complex, which was shown to be localized near the basal membrane of seminiferous tubules in the testes. These findings suggest the possible involvement of angiotensin-II mediated action on these proteins in executing physiological functions as a component of cell-cell junction in the testes.

$\begin{array}{ll}\text { Abbreviations } \\ \text { ARB: } & \text { Angiotensin-II receptor blocker } \\ \text { AT1R: } & \text { Angiotensin type 1 receptor } \\ \text { DAPI: } & 4^{\prime}, 6 \text {-Diamidino-2-phenylindole } \\ \text { DMEM/F12: } & \text { Dulbecco's Modified Eagle Medium/F12 } \\ \text { DNP: } & \text { Dinitrophenylhydrazine } \\ \text { FITC: } & \text { Fluorescein isothiocyanate } \\ \text { GAPDH: } & \text { Glyceraldehyde 3-phosphate } \\ & \text { dehydrogenase } \\ \text { IP: } & \text { Intraperitoneal } \\ \text { RNA: } & \text { Ribonucleic acid } \\ \text { ROS: } & \text { Reactive oxygen species } \\ \text { RT-PCR: } & \text { Reverse-transcriptase polymerase chain } \\ & \text { reaction } \\ \text { SDS: } & \text { Sodium dodecyl sulfate } \\ \text { SOD: } & \text { Superoxide Dismutase } \\ \text { TUNEL: } & \text { Terminal Deoxynucleotidyl } \\ & \text { Transferase-mediated dUTP Nick } \\ & \text { End-labelling. }\end{array}$

\section{Conflict of Interests}

The authors have no conflict of interests to declare.

\section{Acknowledgments}

The authors are grateful to Dr. Yamaguchi and Dr. Okada for providing valuable information about their experiment.

\section{References}

[1] K. Schwarz, M. Simons, J. Reiser et al., "Podocin, a raft-associated component of the glomerular slit diaphragm, interacts with CD2AP and nephrin," Journal of Clinical Investigation, vol. 108, no. 11, pp. 1621-1629, 2001.

[2] T. B. Huber, B. Schermer, R. U. Müller et al., "Podocin and MEC-2 bind cholesterol to regulate the activity of associated ion channels," Proceedings of the National Academy of Sciences of the United States of America, vol. 103, no. 46, pp. 17079-17086, 2006. 
[3] C. Y. Cheng and D. D. Mruk, "The blood-testis barrier and its implications for male contraception," Pharmacological Reviews, vol. 64, no. 1, pp. 16-64, 2012.

[4] K. Chiba, K. Yamaguchi, M. Ando, H. Miyake, and M. Fujisawa, "Expression pattern of testicular claudin-11 in infertile men," Urology, vol. 80, no. 5, pp. 1161.e13-1161.e17, 2012.

[5] K. Okada, H. Miyake, K. Yamaguchi et al., "Critical function of RA-GEF-2/Rapgef6, a guanine nucleotide exchange factor for Rapl, in mouse spermatogenesis," Biochemical and Biophysical Research Communications, vol. 445, no. 1, pp. 89-94, 2014.

[6] M. Relle, H. Cash, C. Brochhausen et al., "New perspectives on the renal slit diaphragm protein podocin," Modern Pathology, vol. 24, no. 8, pp. 1101-1110, 2011.

[7] L. Liu, K. Aya, H. Tanaka, J. Shimizu, S. Ito, and Y. Seino, "Nephrin is an important component of the barrier system in the testis," Acta Medica Okayama, vol. 55, no. 3, pp. 161-165, 2001.

[8] T. Nakatsue, H. Koike, G. D. Han et al., "Nephrin and podocin dissociate at the onset of proteinuria in experimental membranous nephropathy," Kidney International, vol. 67, no. 6, pp. 2239-2253, 2005.

[9] A. Benigni, S. Tomasoni, E. Gagliardini et al., "Blocking angiotensin II synthesis/activity preserves glomerular nephrin in rats with severe nephrosis," Journal of the American Society of Nephrology, vol. 12, no. 5, pp. 941-948, 2001.

[10] S. Okui, H. Yamamoto, W. Li et al., "Cisplatin-induced acute renal failure in mice is mediated by chymase-activated angiotensin-aldosterone system and interleukin-18," European Journal of Pharmacology, vol. 685, no. 1-3, pp. 149-155, 2012.

[11] S. Saleh, A. A. Ain-Shoka, E. El-Demerdash, and M. M. Khalef, "Protective effects of the angiotensin II receptor blocker losartan on cisplatin-induced kidney injury," Chemotherapy, vol. 55, no. 6, pp. 399-406, 2009.

[12] J. L. Alves-Pereira, E. D. C. Frantz, and C. da Fonte Ramos, "Beneficial effects of renin-angiotensin system blockers on testicular steroidogenesis," Journal of Urology, vol. 192, no. 6, pp. 1878-1883, 2014.

[13] S. Kushwaha and G. B. Jena, "Effects of nicotine on the testicular toxicity of streptozotocin-induced diabetic rat: intervention of enalapril," Human and Experimental Toxicology, vol. 33, no. 6, pp. 609-622, 2014.

[14] K. Shiraishi, K.-I. Yoshida, T. Fujimiya, and K. Naito, "Angiotensin II dependent testicular fibrosis and effects on spermatogenesis after vasectomy in the rat," Journal of Urology, vol. 170, no. 5, pp. 2104-2108, 2003.

[15] P. M. Petersen, S. W. Hansen, A. Giwercman, M. Rorth, and N. E. Skakkebaek, "Dose-dependent impairment of testicular function in patients treated with cisplatin-based chemotherapy for germ cell cancer," Annals of Oncology, vol. 5, no. 4, pp. 355358, 1994.

[16] A. Giwercman and P. M. Petersen, "Cancer and male infertility," Best Practice \& Research: Clinical Endocrinology \& Metabolism, vol. 14, no. 3, pp. 453-471, 2000.

[17] L. M. Pogach, Y. Lee, S. Gould, W. Giglio, M. Meyenhofer, and H. F. S. Huang, "Characterization of cis-platinum-induced Sertoli cell dysfunction in rodents," Toxicology and Applied Pharmacology, vol. 98, no. 2, pp. 350-361, 1989.

[18] D. Mohammadnejad, A. Abedelahi, J. Soleimani-rad, A. Mohammadi-roshandeh, M. Rashtbar, and A. Azami, "Degenerative effect of cisplatin on testicular germinal epithelium," Advanced Pharmaceutical Bulletin, vol. 2, no. 2, pp. 173-177, 2012.
[19] National Research Council Committee for the Update of the Guide for the Care and Use of Laboratory Animals, "The national academies collection: reports funded by National Institutes of Health," in Guide for the Care and Use of Laboratory Animals, National Academies Press, Ed., National Academy of Sciences, Washington, DC, USA, 2011.

[20] M. R. Ghassemifar, B. Sheth, T. Papenbrock, H. J. Leese, F. D. Houghton, and T. P. Fleming, "Occludin $\mathrm{TM}^{-}$: an isoform of the tight junction protein present in primates lacking the fourth transmembrane domain," Journal of Cell Science, vol. 115, no. 15, pp. 3171-3180, 2002.

[21] A. Nambu and Y. Kumamoto, "Studies of spermatogenic damages induced by anti-cancer agent and anti-androgenic agents in rat testes," The Japanese Journal of Urology, vol. 86, no. 7, pp. 1221-1230, 1995.

[22] W. Kittikulsuth, J. S. Pollock, and D. M. Pollock, "Loss of renal medullary endothelin B receptor function during salt deprivation is regulated by angiotensin II," The American Journal of Physiology-Renal Physiology, vol. 303, no. 5, pp. F659-F666, 2012.

[23] H. Okada, M. Dobashi, T. Yamazaki et al., "Conventional versus microdissection testicular sperm extraction for nonobstructive azoospermia," Journal of Urology, vol. 168, no. 3, pp. 1063-1067, 2002.

[24] G. Verhoeven, E. Denolet, J. Swinnen et al., "The role of androgens in the control of spermatogenesis: lessons from transgenic models involving a Sertoli cell-selective knockout of the androgen receptor," Animal Reproduction, vol. 4, no. 1-2, pp. 3-14, 2007.

[25] M. C. Leal, S. V. B. Pinheiro, A. J. Ferreira et al., "The role of angiotensin-(1-7) receptor Mas in spermatogenesis in mice and rats," Journal of Anatomy, vol. 214, no. 5, pp. 736-743, 2009.

[26] C. Schneeberger, P. Speiser, F. Kury, and R. Zeillinger, "Quantitative detection of reverse transcriptase-PCR products by means of a novel and sensitive DNA stain," PCR Methods and Applications, vol. 4, no. 4, pp. 234-238, 1995.

[27] H. Kawachi, H. Koike, H. Kurihara, T. Sakai, and F. Shimizu, "Cloning of rat homologue of podocin: expression in proteinuric states and in developing glomeruli," Journal of the American Society of Nephrology, vol. 14, no. 1, pp. 46-56, 2003.

[28] Q. Fan, J. Ding, J. Zhang, N. Guan, and J. Deng, "Effect of the knockdown of podocin mRNA on nephrin and alpha-actinin in mouse podocyte," Experimental Biology and Medicine, vol. 229, no. 9, pp. 964-970, 2004.

[29] C. A. Schneider, W. S. Rasband, and K. W. Eliceiri, "NIH Image to ImageJ: 25 years of image analysis," Nature Methods, vol. 9, no. 7, pp. 671-675, 2012.

[30] T. Sato, K. Seyama, Y. Sato et al., "Senescence marker protein-30 protects mice lungs from oxidative stress, aging, and smoking," American Journal of Respiratory and Critical Care Medicine, vol. 174, no. 5, pp. 530-537, 2006.

[31] B. I. Frohnert and D. A. Bernlohr, "Protein carbonylation, mitochondrial dysfunction, and insulin resistance," Advances in Nutrition, vol. 4, no. 2, pp. 157-163, 2013.

[32] H.-H. Parving, H. Lehnert, J. Brochner-Mortensen, R. Gomis, S. Andersen, and P. Arner, "The effect of irbesartan on the development of diabetic nephropathy in patients with type 2 diabetes," The New England Journal of Medicine, vol. 345, no. 12, pp. 870-878, 2001.

[33] W. Lin, W. Ye, L. Cai et al., "The roles of multiple importins for nuclear import of murine aristaless-related homeobox protein," 
The Journal of Biological Chemistry, vol. 284, no. 30, pp. 2042820439, 2009.

[34] E. Schnabel, J. M. Anderson, and M. G. Farquhar, "The tight junction protein ZO-1 is concentrated along slit diaphragms of the glomerular epithelium," Journal of Cell Biology, vol. 111, no. 3, pp. 1255-1263, 1990.

[35] H. Kawachi, N. Miyauchi, K. Suzuki, D. H. Gi, M. Orikasa, and F. Shimizu, "Role of podocyte slit diaphragm as a filtration barrier," Nephrology, vol. 11, no. 4, pp. 274-281, 2006.

[36] L. M. Domke, S. Rickelt, Y. Dörflinger et al., "The cell-cell junctions of mammalian testes: I. The adhering junctions of the seminiferous epithelium represent special differentiation structures," Cell and Tissue Research, vol. 357, no. 3, pp. 645-665, 2014.

[37] S. Gotoh, M. Mine, K. Ishizaka, H. Kaneoya, and M. Yokokawa, "Changes in lanthanum permeability of rat Sertoli cell tight junction after CDDP administration," The Japanese Journal of Urology, vol. 81, no. 1, pp. 45-48, 1990.

[38] F. Bonnet, M. E. Cooper, H. Kawachi, T. J. Allen, G. Boner, and Z. Cao, "Irbesartan normalises the deficiency in glomerular nephrin expression in a model of diabetes and hypertension," Diabetologia, vol. 44, no. 7, pp. 874-877, 2001.

[39] T. B. Huber, M. Köttgen, B. Schilling, G. Walz, and T. Benzing, "Interaction with podocin facilitates nephrin signaling," Journal of Biological Chemistry, vol. 276, no. 45, pp. 41543-41546, 2001.

[40] B. S. Brooke, J. P. Habashi, D. P. Judge, N. Patel, B. Loeys, and H. C. Dietz III, "Angiotensin II blockade and aortic-root dilation in Marfan's syndrome," The New England Journal of Medicine, vol. 358, no. 26, pp. 2787-2795, 2008.

[41] S. Fuchs, K. Frenzel, C. Hubert et al., "Male fertility is dependent on dipeptidase activity of testis ACE," Nature Medicine, vol. 11, no. 11, pp. 1140-1142, 2005.

[42] T. Ichiki, "Angiotensin II accelerates vascular inflammation and atherosclerosis," The Journal of Japanese College of Angiology, vol. 49, no. 4, pp. 287-292, 2009.

[43] K. K. Griendling, C. A. Minieri, J. D. Ollerenshaw, and R. W. Alexander, "Angiotensin II stimulates NADH and NADPH oxidase activity in cultured vascular smooth muscle cells," Circulation Research, vol. 74, no. 6, pp. 1141-1148, 1994.

[44] R. John Aitken and S. D. Roman, "Antioxidant systems and oxidative stress in the testes," Advances in Experimental Medicine and Biology, vol. 636, pp. 153-171, 2008.

[45] N. Kilarkaje, A. M. Mousa, M. M. Al-Bader, and K. M. Khan, "Antioxidants enhance the recovery of three cycles of bleomycin, etoposide, and cisplatin-induced testicular dysfunction, pituitary-testicular axis, and fertility in rats," Fertility and Sterility, vol. 100, no. 4, pp. 1151.e5-1159.e5, 2013.

[46] H. Anand, M. M. Misro, S. B. Sharma, and S. Prakash, "Protective effects of Eugenia jambolana extract versus $\mathrm{N}$-acetyl cysteine against cisplatin-induced damage in rat testis," Andrologia, vol. 47, no. 2, pp. 194-208, 2015.

[47] K. Narayana, M. Al-Bader, A. Mousa, and K. M. Khan, "Molecular effects of chemotherapeutic drugs and their modulation by antioxidants in the testis," European Journal of Pharmacology, vol. 674, no. 2-3, pp. 207-216, 2012.

[48] E. A. Salem, N. A. Salem, A. M. Maarouf, E. C. Serefoglu, and W. J. G. Hellstrom, "Selenium and lycopene attenuate cisplatininduced testicular toxicity associated with oxidative stress in Wistar rats," Urology, vol. 79, no. 5, pp. 1184.e1-1184.e6, 2012. 


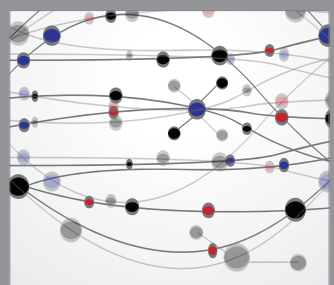

The Scientific World Journal
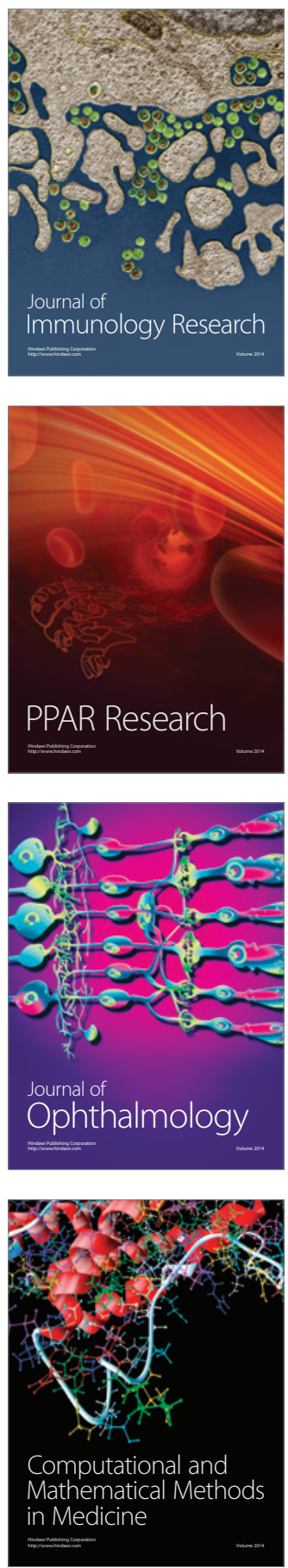

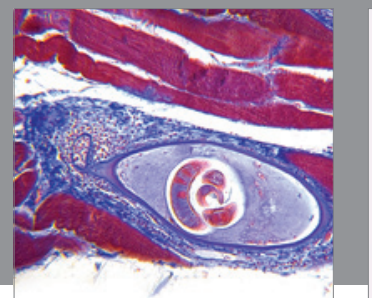

Gastroenterology

Research and Practice
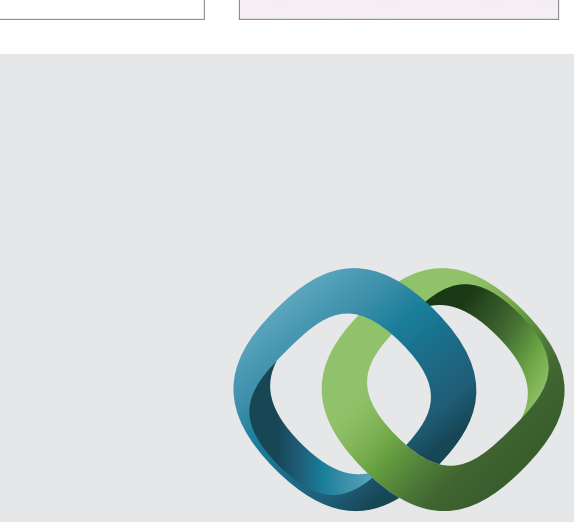

\section{Hindawi}

Submit your manuscripts at

http://www.hindawi.com
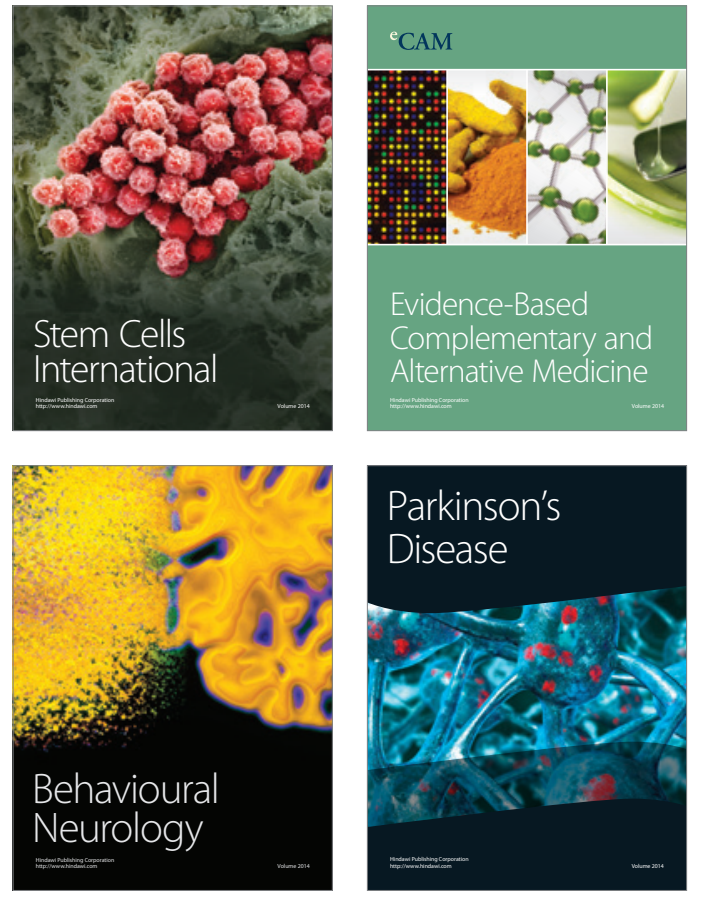
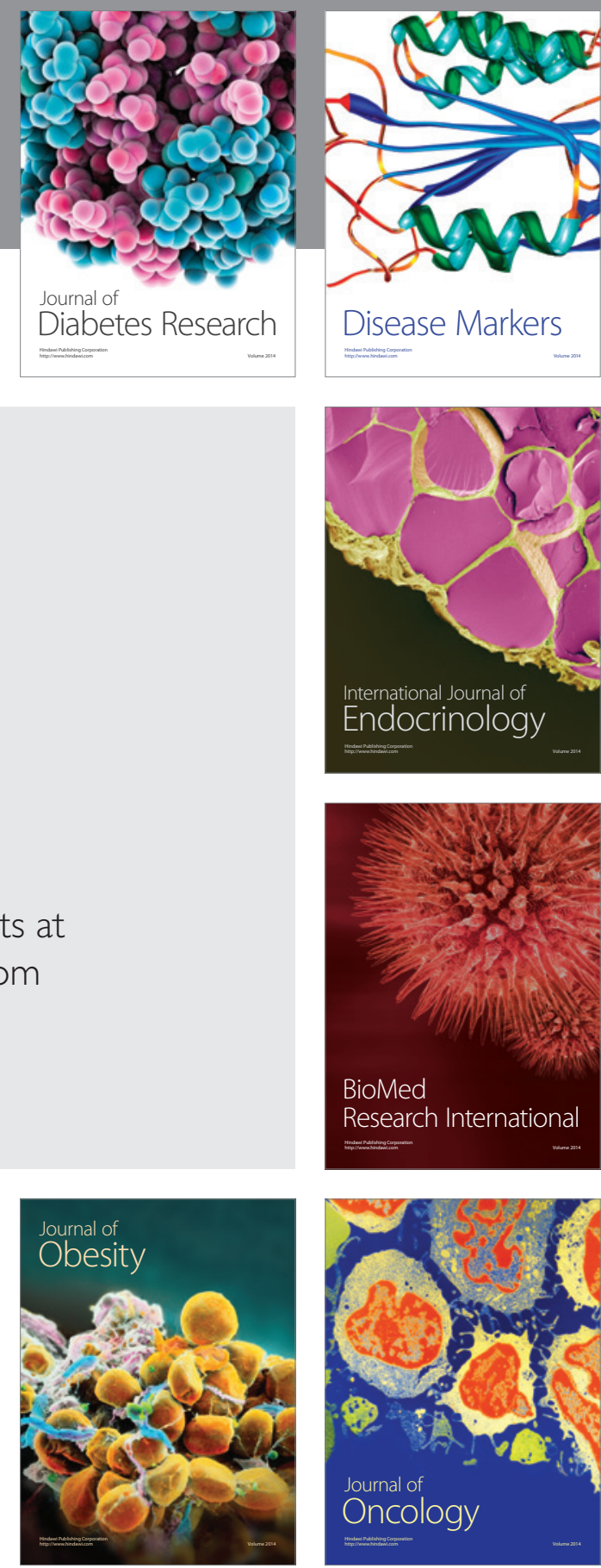

Disease Markers
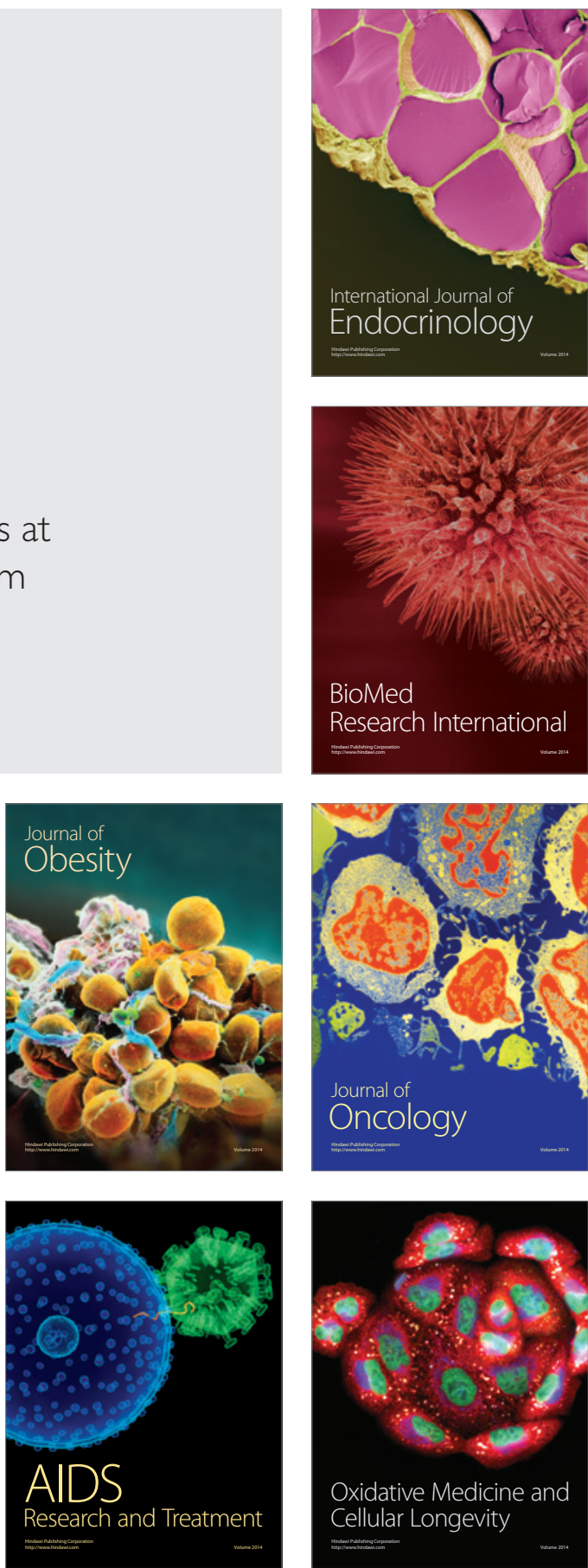